\title{
COMMUTING ANALYTIC FUNCTIONS WITHOUT FIXED POINTS
}

\author{
DONALD F. BEHAN
}

\begin{abstract}
Let $A$ be the set of nonidentity analytic functions which map the open unit disk into itself. Wolff has shown that the iterates of $f \in A$ converge uniformly on compact sets to a constant $T(f)$, unless $f$ is an elliptic conformal automorphism of the disk. This paper presents a proof that if $f$ and $g$ are in $A$ and commute under composition, and if $f$ is not a hyperbolic conformal automorphism of the disk, then $T(f)=T(g)$. This extends, in a sense, a result of Shields. The proof involves the so-called angular derivative of a function in $A$ at a boundary point of the disk.
\end{abstract}

Let $D$ be the open unit disk in the complex plane. Let $\bar{D}$ be its closure. Shields [5] has proved the following result.

THEOREM 1. If $f$ and $g$ are continuous in $\bar{D}$, analytic in $D$, and map $\bar{D}$ into itself, and if $f \circ g=g \circ f$, then $f$ and $g$ have a common fixed point.

Let $A$ be the set of all analytic functions which map $D$ into $D$, except for the identity function, which we exclude. This paper presents an extension of the result of Shields to the set $A$.

For $f \in A$ we define the iterates of $f$ recursively by $f^{1}=f$, and $f^{n+1}=$ $f \circ f^{n}$ when $n \in Z^{+}$. A member of $A$ which maps $D$ univalently onto $D$ will be called a conformal automorphism (c.a.) of $D$. We shall assume that the reader is acquainted with the standard classification of linear fractional transformations as elliptic, hyperbolic, parabolic, or loxodromic, as given in [3, p. 70]. Each c.a. of $D$ is of one of the first three types mentioned. The elliptic transformations yield noneuclidean rotations of $D$ with the hyperbolic metric, while the hyperbolic and parabolic transformations have their fixed points on the boundary of $D$.

THEOREM 2 (WOLFF [7]). If $f \in A$ is not an elliptic c.a. of $D$, then there is a constant $T(f) \in \bar{D}$ for which $\lim _{n \rightarrow \infty} f^{n}=T(f)$ uniformly on compact sets.

Presented to the Society, January 24, 1971; received by the editors March 1, 1971.

AMS (MOS) subject classifications (1970). Primary 30A20, 30A76; Secondary 39A15, 30A72.

Key words and phrases. Commuting under composition, iteration, fixed point, angular derivative, Julia lemma, chain rule, Lindelöf theorem.

(c) American Mathematical Society 1973 
If $T(f)$ is in $D$, then it is a fixed point of $f$; and if $f$ has a fixed point in $D$, it has only one, and that fixed point is $T(f)$. The preceding sentence is still true if we extend the definition of $T$ so that when $f$ is an elliptic c.a. of $D$, then $T(f)$ is the fixed point of $f$ in $D$.

Suppose that $f$ and $g$ are in $A$, that $T(f) \in D$, and that $f \circ g=g \circ f$. Then

$$
g[T(f)]=g(f[T(f)])=f(g[T(f)]),
$$

so $g[T(f)]$ is a fixed point for $f$. This implies $T(f)=g[T(f)]$, and consequently $T(g)=T(f)$. We wish to extend this result, as far as possible, to functions $f$ for which $T(f) \notin D$. If two linear fractional transformations have the same set of fixed points, then they commute $[3, p .72]$. There exist pairs $f, g$ of hyperbolic conformal automorphisms of $D$ which have a common set of fixed points, but for which $T(f) \neq T(g)$. For example

$$
f(z)=\left(z-\frac{1}{2}\right) /\left(1-\frac{1}{2} z\right) \text { and } g(z)=\left(z+\frac{1}{2}\right) /\left(1+\frac{1}{2} z\right)
$$

defines such a pair. Thus it is not true in general that commuting members of $A$ satisfy $T(f)=T(g)$, but we shall show that the only exceptional cases involve pairs of hyperbolic conformal automorphisms of $D$.

The only cases in which linear fractional transformations commute without having the same fixed points involve certain pairs of elliptic transformations which cannot occur as pairs in $A$. Thus two conformal automorphisms of $D$ commute if and only if they have the same fixed points.

For ease of notation when $f \in A$ and $|\zeta|=1$ we shall write $f(\zeta)=\eta$ to mean $\lim _{r \rightarrow 1^{-}} f(r \zeta)=\eta$, and in that case we write $f^{\prime}(\zeta)=u$ to mean

$$
\lim _{r \rightarrow 1^{-}} \frac{f(r \zeta)-\eta}{r \zeta-\zeta}=u
$$

We shall have several occasions to use Julia's lemma, which we state in a form which is essentially that of $[4$, p. 58].

THEOREM 3. If $|\zeta|=|\eta|=1$, and $f \in A$ satisfies $f(\zeta)=\eta$, then there is an extended real number $c$, with $0<c \leqq \infty$, such that

$$
\lim _{z \rightarrow \zeta}\left|\frac{f(z)-\eta}{z-\zeta}\right|=c
$$

as $z$ approaches $\zeta$ through any open triangle in $D$ with vertex at $\zeta$. If $c$ is finite then $f^{\prime}(\zeta)=c \eta / \zeta$.

If $c$ is finite, then it is also true that $\lim _{z \rightarrow \zeta} f^{\prime}(z)=f^{\prime}(\zeta)$ when $z$ approaches $\zeta$ as in Theorem 3. 
If $f \in A$ and $|\zeta|=1$, then $T(f)=\zeta$ if and only if $f(\zeta)=\zeta$ and $f^{\prime}(\zeta) \leqq 1$. (See [6] for example.)

We shall use the following result of Lindelöf [2, p. 19].

THEOREM 4. Let $f$ be analytic and bounded in D. If $f(z)$ approaches $w$ as $z$ approaches $e^{i \theta}$ along some curve $\gamma$ lying in $D$ except for its terminal point at $e^{i \theta}$, then $f(z)$ approaches $w$ uniformly as $z$ approaches $e^{i \theta}$ in any open triangle in $D$ with vertex $e^{i \theta}$.

LEMMA 5. Suppose $f$ and $g$ are in $A$, and that $f \circ g=g \circ f$. Suppose that $T(g)=\zeta$ where $|\zeta|=1$. Then $f(\zeta)=\zeta$.

Proof. We have $g(0) \in D$, and by the uniqueness of $T(g)$ we have $g(0) \neq 0$. Let $S$ be the segment from 0 to $g(0)$. Then, roughly, what we propose to do is to form a curve $\gamma$ by joining successive images of $S$ under $g^{n}$, and then to use commutativity to show that $f \circ \gamma$ approaches $\zeta$.

For $0 \leqq t<1$ let $n(t)$ be the greatest integer less than or equal to $-\log _{2}(1-t)$. Let $w=g(0)$. Then for $0 \leqq t<1$ we define

$$
\gamma(t)=g^{n(t)}\left[2^{n(t)+1} t w-\left(2^{n(t)+1}-2\right) w\right]
$$

and we define $\gamma(1)=\zeta$. It is clear that $\gamma$ is continuous for $2^{-n-1}<1-t<2^{-n}$, with $n$ a nonnegative integer. We find that $\lim \gamma(t)=g^{n}(0)=\gamma\left(1-2^{-n}\right)$ as $t$ approaches $1-2^{-n}$ from above, and that $\lim \gamma(t)=g^{n-1}(w)=g^{n}(0)$ as $t$ approaches $1-2^{-n}$ from below. Thus $\gamma$ is continuous, except possibly at 1 . But $\lim _{n \rightarrow \infty} g^{n}=\zeta$ uniformly on $S$, and therefore $\lim _{t \rightarrow 1} \gamma(t)=\zeta$, so $\gamma$ is continuous and terminates at $\zeta$.

Since $f(S)$ is compact, $g^{n}$ approaches $\zeta$ uniformly on $f(S)$. Since $f\left[g^{n}(S)\right]=g^{n}[f(S)]$, given any neighborhood $U$ of $\zeta, f\left[g^{n}(S)\right] \subset U$ for large $n$. Thus $\lim _{t \rightarrow 1} f[\gamma(t)]=\zeta$. By Lindelöf's theorem $\lim _{r \rightarrow 1^{-}} f(r \zeta)=\zeta$, which we write $f(\zeta)=\zeta$.

THEOREM 6. If $f \in A$ is not a hyperbolic c.a. of $D$, and if $g \in A$ satisfies $f \circ g=g \circ f$, then $T(f)=T(g)$.

The proof will use a sequence of lemmas.

Lemma 7 (ChaIN Rule). Suppose that $f$ and $g$ are in $A$, that $|\zeta|=|\eta|=$ $|\tau|=1$, that $f(\zeta)=\eta$, and $g(\eta)=\tau$. Then

$$
\lim _{r \rightarrow 1^{-}} \frac{g[f(r \zeta)]-\tau}{r \zeta-\zeta}=g^{\prime}(\eta) f^{\prime}(\zeta) .
$$

Proof. Without loss of generality we take $\zeta=\eta=\tau=1$.

If $f^{\prime}(1)$ and $g^{\prime}(1)$ are both finite, then $f(r)$ approaches 1 nontangentially, and by Julia's lemma $\lim _{r \rightarrow 1^{-}} f^{\prime}(r)=f^{\prime}(1)$ and $\lim _{z \rightarrow 1} g^{\prime}(z)=g^{\prime}(1)$ when $z$ 
approaches 1 nontangentially. The desired result follows from the chain rule for ordinary derivatives. For $r \in D$ real,

$$
\left|\frac{1-g[f(r)]}{1-r}\right| \geqq \frac{1-|g[f(r)]|}{1-r}=\frac{1-|g[f(r)]|}{1-|f(r)|} \cdot \frac{1-|f(r)|}{1-r} .
$$

For all $h \in A[\mathbf{1}$, p. 25],

$$
\frac{1-|h(z)|}{1-|z|} \geqq \frac{1-|h(0)|}{1+|h(0)|}>0
$$

for all $z \in D$, so each factor in the last term of (1) has a positive infimum. It is also true [1, p. 27] that if $h^{\prime}(1)=\infty$, then

$$
\lim _{z \rightarrow 1} \frac{1-|h(z)|}{1-|z|}=\infty
$$

when $z$ approaches 1 in such a way that $h(z)$ approaches 1 .

If $f^{\prime}(1)=\infty$, the desired result follows easily.

Suppose $g^{\prime}(1)=\infty$ and $f^{\prime}(1) \neq \infty$. Then $f(r)$ approaches 1 nontangentially, so the first factor in the last term of (1) approaches $\infty$. Since the second factor has a positive infimum, the desired result follows. This completes the proof of Lemma 7.

As a consequence of Lemma 7, we see that if $f$ and $g$ in $A$ satisfy $f \circ g=$ $g \circ f$ and $|T(f)|=1$, then $T\left(f^{n} \circ g^{m}\right)$ is $T(f)$ or $T(g)$. For if $T\left(f^{n} \circ g^{m}\right)=\zeta$, then since $f^{n} \circ g^{m}$ commutes with $f$, we have $|\zeta|=1$, and Lemma 5 shows that $f(\zeta)=g(\zeta)=\zeta$. The derivative of $f^{n} \circ g^{m}$ at $\zeta$ is $\left(f^{\prime}(\zeta)\right)^{n}\left(g^{\prime}(\zeta)\right)^{m}$ by Lemma 7. In order for this product to be less than or equal to 1 , it is necessary that $f^{\prime}(\zeta) \leqq 1$ or $g^{\prime}(\zeta) \leqq 1$, which implies $\zeta=T(f)$ or $\zeta=T(g)$.

We shall use the following lemma for the special case $\theta=\phi$.

LEMMA 8. If $f \in A, f\left(e^{i \theta_{1}}\right)=e^{i \phi_{1}}$, and $f\left(e^{i \theta_{2}}\right)=e^{i \phi_{2}}$, where $\theta=\theta_{2}-\theta_{1} \neq 0$ $(\bmod 2 \pi)$ and $\phi=\phi_{2}-\phi_{1} \neq 0(\bmod 2 \pi)$, then

$$
\left|f^{\prime}\left(e^{i \theta_{1}}\right) f^{\prime}\left(e^{i \theta_{2}}\right)\right| \geqq\left[\frac{\sin \phi / 2}{\sin \theta / 2}\right]^{2} .
$$

This inequality is best possible, and equality obtains only for conformal automorphisms of $D$.

Proof. We suppose without loss of generality that $\theta_{1}=\phi_{1}=0$. Let $\zeta=e^{i \phi}$ and $\eta=e^{i \theta}$. The set $U$ of conformal automorphisms $h$ of $D$ such that $h(1)=1$ and $h(\zeta)=\eta$ is nonempty, and each such $h$ satisfies $f \circ h(1)=1$ and $f \circ h(\zeta)=\zeta$. By the uniqueness of $T(f \circ h)$ it follows that

$$
\left|f^{\prime}(\eta) h^{\prime}(\zeta)\right|>1 \text { or }\left|f^{\prime}(1) h^{\prime}(1)\right|>1
$$


unless $f \circ h$ is the identity, in which case $f$ is a conformal automorphism of $D$. We shall show that (3) implies

$$
\left|f^{\prime}(1) f^{\prime}(\eta)\right|>1 /\left|h^{\prime}(1) h^{\prime}(\zeta)\right| .
$$

We observe that the value of $h^{\prime}(1) h^{\prime}(\zeta)$ does not depend on $h \in U$, for if $h_{1}$ and $h_{2}$ are in $U$, then $h_{1} h_{2}^{-1}$ has fixed points 1 and $\eta$. Therefore the derivative of $h_{1} h_{2}^{-1}$ at $\eta$ is the reciprocal of its derivative at 1 , and this yields

When $|a|<1$ let

$$
h_{1}^{\prime}(1) h_{1}^{\prime}(\zeta) / h_{2}^{\prime}(1) h_{2}^{\prime}(\zeta)=1
$$

$$
h_{a}(z)=\frac{1-\bar{a}}{1-a} \frac{z-a}{1-\bar{a} z} .
$$

Then

$$
h_{a}^{\prime}(1)=\frac{1-|a|^{2}}{(1-a)(1-\bar{a})}
$$

which is a continuous function of $a$ for $a \in D$. We have $\lim _{a \rightarrow 1} h_{a}^{\prime}(1)=\infty$ and $\lim _{a \rightarrow w} h_{a}^{\prime}(1)=0$ when $|w|=1$ but $w \neq 1$. Moreover $h_{a} \in U$ provided that

$$
\arg \left(e^{i \phi}-a\right)-\arg (1-a)=(\phi+\theta) / 2(\bmod 2 \pi) .
$$

The values of $a$ in $D$ which satisfy (4) form a circular arc or line segment terminating at 1 and $e^{i \phi}$. Since this is a connected set, $\left|h_{a}^{\prime}(1)\right|$ takes all positive real values for $h_{a} \in U$. that

If it were true that $\left|f^{\prime}(1) f^{\prime}(\eta)\right|<1 /\left|h_{a}^{\prime}(1) h_{a}^{\prime}(\zeta)\right|$, then it would follow

$$
\left|h_{a}^{\prime}(1) h_{a}^{\prime}(\zeta)\right| \cdot\left|f^{\prime}(\eta)\right|<1 /\left|f^{\prime}(1)\right|,
$$

and since $h_{a}^{\prime}(1) h_{a}^{\prime}(\zeta)$ is independent of $a$, we could choose $a$ so that

$$
\left|h_{a}^{\prime}(1) h_{a}^{\prime}(\zeta)\right| \cdot\left|f^{\prime}(\eta)\right|<\left|h_{a}^{\prime}(1)\right|<1 /\left|f^{\prime}(1)\right| .
$$

But then we would have

$$
\left|f^{\prime}(\eta) h_{a}^{\prime}(\zeta)\right|<1 \text { and }\left|f^{\prime}(1) h_{a}^{\prime}(1)\right|<1,
$$

which contradicts (3).

We find that a permissible value of $a$ is

$$
\frac{\sin (\theta-\phi) / 4}{\sin (\theta+\phi) / 4} e^{i \phi / 2} \text {. }
$$

A computation using the observation that $|1-a \zeta|=|1-a|$ and the cosine 
rule yields

$$
\frac{1}{\left|h_{a}^{\prime}(1) h_{a}^{\prime}(\zeta)\right|}=\left[\frac{\sin \phi / 2}{\sin \theta / 2}\right]^{2} \text {. }
$$

This completes the proof of (2).

If $\left|f^{\prime}(1) f^{\prime}(\eta)\right|=1 /\left|h_{a}^{\prime}(1) h_{a}^{\prime}(\zeta)\right|$, then there exists $h \in U$ such that $1 /\left|h^{\prime}(1)\right|=\left|f^{\prime}(1)\right|$. Then $\left|f^{\prime}(1) h^{\prime}(1)\right|=1$ and $\left|f^{\prime}(\eta) h^{\prime}(\zeta)\right|=1$, so $f=h^{-1}$ is a c.a. of $D$. If $f$ is a c.a. of $D$, which satisfies the hypotheses of Lemma 8 , then equality holds in (2). This completes the proof of Lemma 8.

We see, in particular, that if $f \in A, f\left(e^{i \theta_{1}}\right)=e^{i \theta_{1}}$, and $f\left(e^{i \theta_{2}}\right)=e^{i \theta_{2}}$, then

$$
\left|f^{\prime}\left(e^{i \theta_{1}}\right) f^{\prime}\left(e^{i \theta_{2}}\right)\right| \geqq 1
$$

with equality only if $f$ is a (hyperbolic) c.a. of $D$.

If the condition $\phi \neq 0$ in Lemma 8 is omitted, then the infimum of the left-hand side of (2) for $f \in A$ is 0 .

LEMMA 9. Given $f^{\prime}(\zeta) f^{\prime}(\eta)>1, g^{\prime}(\zeta) g^{\prime}(\eta) \geqq 1, f^{\prime}(\zeta) \leqq 1, f^{\prime}(\eta)>1$, $g^{\prime}(\zeta)>1, g^{\prime}(\eta) \leqq 1$, all positive or infinite, there are positive integers $n$ and $m$ such that $\left(f^{\prime}(\zeta)\right)^{n}\left(g^{\prime}(\zeta)\right)^{m}>1$ and $\left(f^{\prime}(\eta)\right)^{n}\left(g^{\prime}(\eta)\right)^{m}>1$.

(In order to simplify notation we write $\infty>1$.)

Proof. By taking logarithms one obtains the following equivalent statement.

Given $a+b>0, c+d \geqq 0, a \leqq 0, b>0, c>0, d \leqq 0$, there are positive integers $n$ and $m$ such that $n a+m c>0$ and $n b+m d>0$. We prove this form of the statement.

If $d=0$ we simply take $n=1$ and $m$ large. Suppose $d \neq 0$. We find that $a+c|a| c \mid=0$. Therefore

$$
0<(a+b)+(c+d)|a| c|=b+d| a|c|,
$$

hence $|a| c|<-b| d$, so that $|a| c|<| b|d|$. Let $n$ and $m$ be positive integers such that

Then

$$
|a| c|<m| n<|b / d| \text {. }
$$

and

$$
n a+m c=n(a+(m / n) c)>n(a+|a / c| c)=0
$$

$$
n b+m d=n(b+(m / n) d)>n(b+|b / d| d)=0 .
$$

Proof of Theorem 6. The only case in question is that in which $T(f)$ and $T(g)$ are of modulus 1 . Assuming that $f$ is not a hyperbolic c.a. of $D$, that $f \circ g=g \circ f$, and that $T(f) \neq T(g)$, we shall arrive at a contradiction.

Let $T(f)=\zeta$ and $T(g)=\eta$. Since $f$ is not a hyperbolic c.a. of $D$, and since $f(\eta)=\eta$, Lemma 8 yields $f^{\prime}(\zeta) f^{\prime}(\eta)>1$ and $g^{\prime}(\zeta) g^{\prime}(\eta) \geqq 1$. We also 
have $f^{\prime}(\zeta) \leqq 1, f^{\prime}(\eta)>1, g^{\prime}(\zeta)>1$, and $g^{\prime}(\eta) \leqq 1$. Now let $n$ and $m$ be as in Lemma 9. Then $T\left(f^{n} \circ g^{m}\right) \neq \zeta$ and $T\left(f^{n} \circ g^{m}\right) \neq \eta$, but as observed following the proof of Lemma 7,T( $\left.f^{n} \circ g^{m}\right)$ must be $\zeta$ or $\eta$. We have arrived at a contradiction, and therefore $T(f)=T(g)$.

\section{REFERENCES}

1. C. Carathéodory, Theory of functions of a complex variable. Vol. 2, 2nd ed., Chelsea, New York, 1960. MR 16, 346; 12, 248.

2. E. F. Collingwood and A. J. Lohwater, The theory of cluster sets, Cambridge Tracts in Math. and Math. Phys., no. 56, Cambridge Univ. Press, Cambridge, 1966. MR 38 \#325.

3. J. Lehner, Discontinuous groups and automorphic functions, Math. Surveys, no. 8, Amer. Math. Soc., Providence, R.I., 1964. MR 29 \#1332.

4. R. Nevanlinna, Eindeutige analytische Funktionen, 2nd ed., Die Grundlehren der math. Wissenschaften, Band 46, Springer-Verlag, Berlin, 1953. MR 15, 208.

5. A. L. Shields, On fixed points of commuting analytic functions, Proc. Amer. Math. Soc. 15 (1964), 703-706. MR 29 \#2790.

6. G. Valiron, Sur l'itération des fonctions holomorphes dans un demi-plan, Bull. Sci. Math. 55 (1931), fasc. 1, 105-128.

7. J. Wolff, Sur l'itération des fonctions bornées, C. R. Acad. Sci. Paris (1926), 200 201.

Department of Mathematics, Union College, Schenectady, New York 12308

Current address: Farm Family Life Insurance Co., Box 656, Albany, New York 12201 